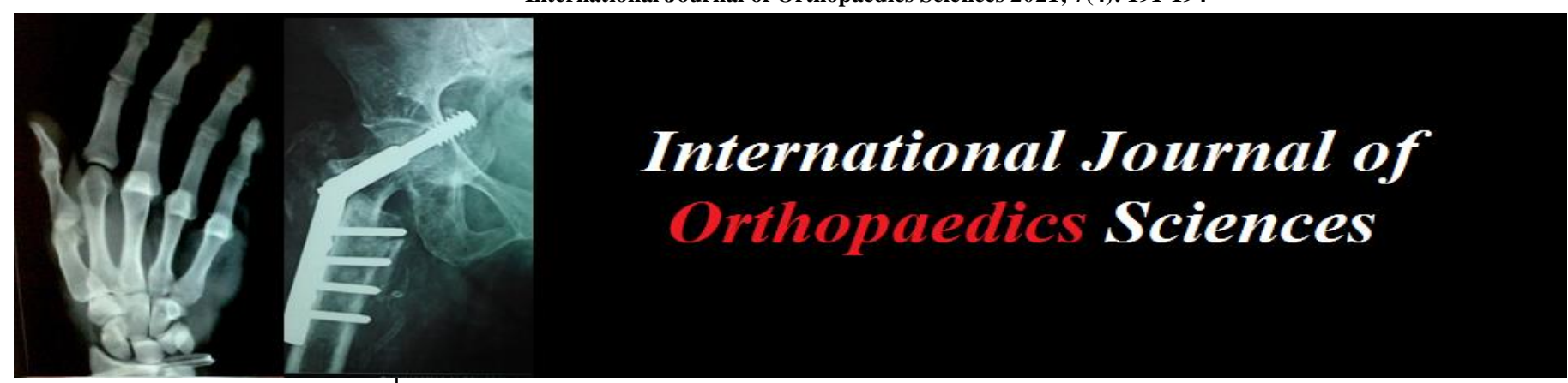

E-ISSN: 2395-1958

P-ISSN: 2706-6630

IJOS 2021; 7(4): 191-194

(C) 2021 IJOS

www.orthopaper.com

Received: 25-06-2021

Accepted: 27-07-2021

Dr. Gnanaprakash Palaniappan Assistant Professor, Department of Orthopedics, PSGIMSR, Coimbatore, Tamil Nadu, India

Dr. Chetan John Rasquinha Assistant Professor, Department of Orthopaedics, PSGIMSR, Coimbatore, Tamil Nadu, India

Dr. Major K Kamlanathan Professor, Department of Orthopaedics, PSGIMSR, Coimbatore, Tamil Nadu, India

Corresponding Author: Dr. Chetan John Rasquinha Assistant Professor, Department of Orthopaedics, PSGIMSR, Coimbatore, Tamil Nadu, India

\section{Critical care challenges in the orthopedic patients: A study}

\section{Dr. Gnanaprakash Palaniappan, Dr. Chetan John Rasquinha and Dr. Major K Kamlanathan}

DOI: https://doi.org/10.22271/ortho.2021.v7.i4c.2884

Abstract

Aim: The purpose of this study is to evaluate the causes, numbers, outcomes of Orthopedic patients requiring intensive care.

Methods: A retrospective and prospective study of patients admitted as in patients, under orthopaedic care, in PGSIMSR, and who required intensive medical care were included in this study.

Conclusion: With increasing trauma and polytrauma cases, coupled with increasing geriatric population, intensive care has becoming more and more common in orthopaedic patients. Mortality rate is higher in the trauma group. Elderly patients are more likely to develop complications due to associated comorbidities. This emphasizes the importance of pre operative and postoperative monitoring of these vulnerable patients.

Keywords: Critical care challenges, orthopedic patients, intertrochanteric fracture

\section{Introduction}

Orthopaedic patients, both trauma and elective orthopedic surgery patients form a significant proportion of patients requiring intensive care and are vulnerable to a wide range of complications, especially the geriatric age group ${ }^{[1]}$. Management of these complications require understanding of preoperative conditions, intraoperative managements and early recognition of any signs and symptoms of any postoperative complications ${ }^{[2,3]}$.

Very few Indian studies have been made about the life threatening medical emergencies in the orthopedic patients requiring intensive care. With increase in geriatric population in India, patients requiring a surgical management like arthroplasties for degenerative disorders is also on the increase. This geriatric population is vulnerable to perioperative and postoperative complications due to their comorbidities. Also due to high velocity polytrauma injuries on the increase the number, the incidence of ICU admissions are also increasing ${ }^{[3]}$. This study will help in a better understanding of such conditions and will help in a better outcome for these patients.

\section{Materials and methods}

A retrospective and prospective study of patients admitted as in patients, under orthopaedic care, in PGSIMSR, and who required intensive medical care were included in this study.

72 patients who were admitted under orthopedic care and subsequently developed problems leading to intensive care were studied. Out of this 42 were male patients and 30 were female patients.

The patients were categorized as trauma patients, polytrauma patients, and elective orthopedic patients.

The study inputs included demographics, reason for admission and orthopedic procedure, problems leading to ICU admissions, and mortality.

\section{Results}

The largest group of ICU admissions were from the trauma and polytrauma group. They accounted for 41 patients-which is about 56 percent. (Fig 1.) 
In this hip fractures-fracture neck of femur and intertrochanteric fracture accounted for the majority-20 patients, which accounts to about 50 percent. 9 patients were of Lower limb fractures, 7 patients were of polytrauma group, 3 patients were upper limb fracture, and two patients were infected hip arthroplaties. (Fig 2.) In the polytrauma group, 4 were from RTA, and three was from fall from height.

In the reasons for ICU admissions, 12 were admitted for close monitoring due to electrolyte imbalances, 5 patients due to acute kidney injury, 10 patients due to respiratory failure, 14 due to need of inotropic support. (Fig 3)

Age wise the average age was 62 years, thus indicating that older trauma patients were more vulnerable due to associated comorbidities and decreased reserve.

In the elective surgery group of 31 patients, the largest was from those requiring total hip replacments-18, total knee replacements were 7 patients, spine surgery were 6 patients (Fig 4, 5)

In the reasons for ICU admissions, 6 were admitted for close monitoring due to electrolyte imbalances, 6 patients due to acute kidney injury, 9 patients due to respiratory failure, 7 due to need of inotropic support and 3 due to DVT. (Fig 6) Age wise, the average age was 67 , again showing that the elederly are vulnerable for developing complications.

In both the groups, hyponatremia -12 patients, hypokalemia-4 and hyperkalemia -2 accounted were recorded.

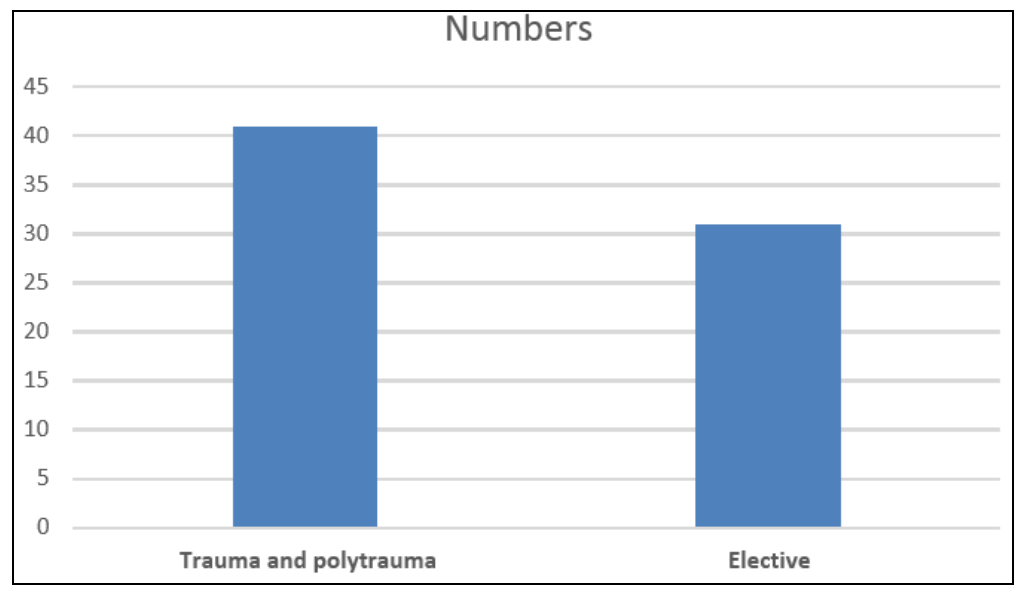

Fig 1: Orthopaedic patients requiring ICU admissions

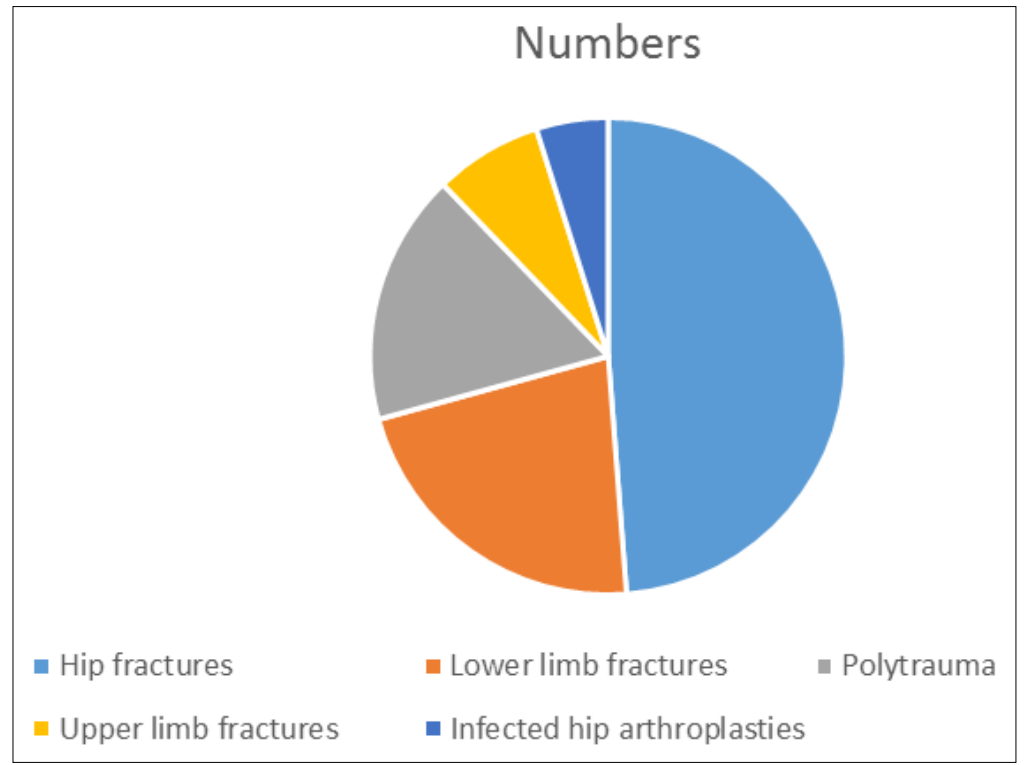

Fig 2: Initial diagnosis in trauma group

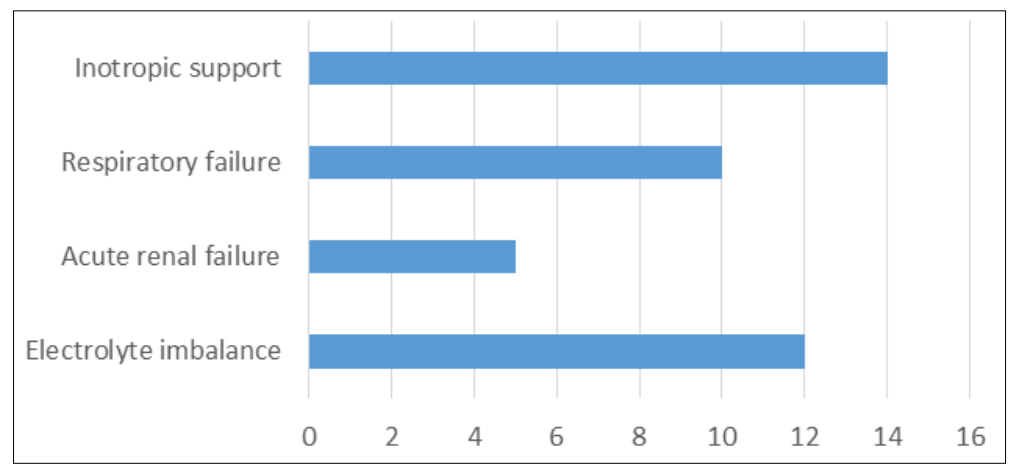

Fig 3: Reasons for admissions in trauma group 


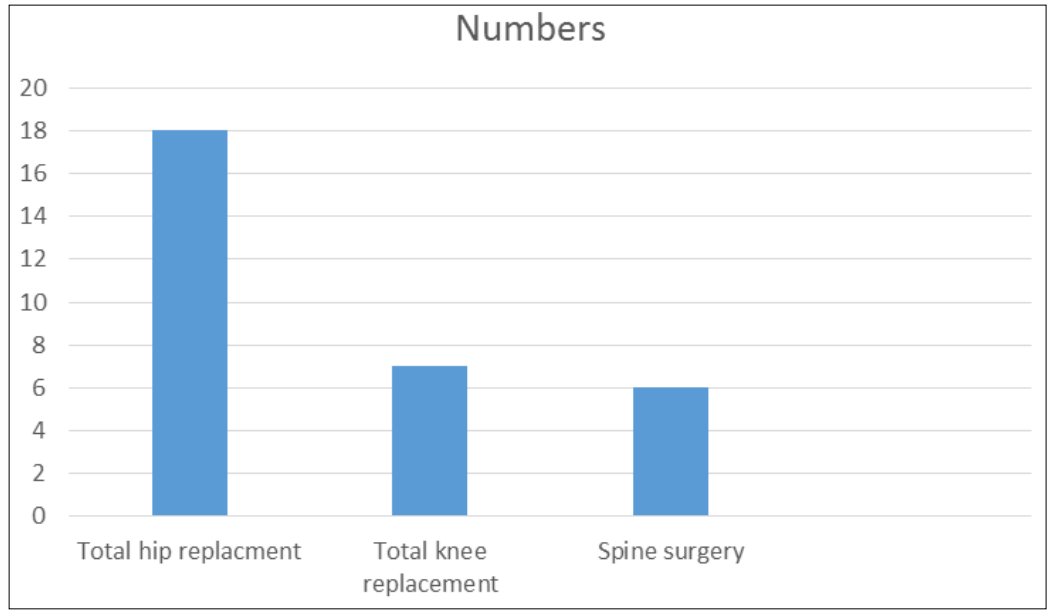

Fig 4: Elective cases initial diagnosis.

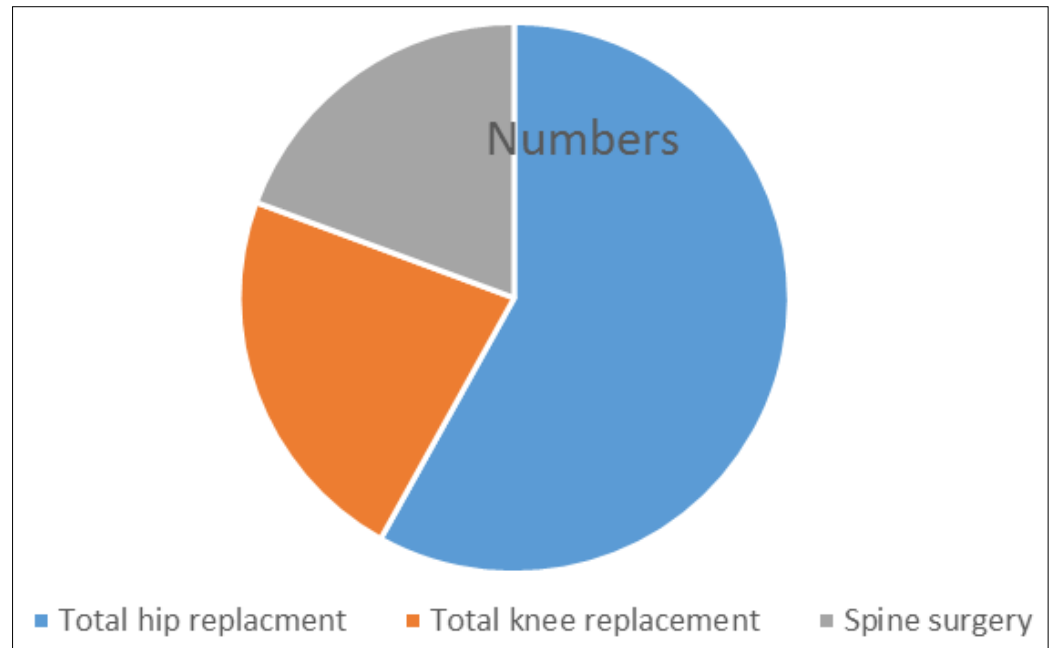

Fig 5: Elective cases initial diagnosis

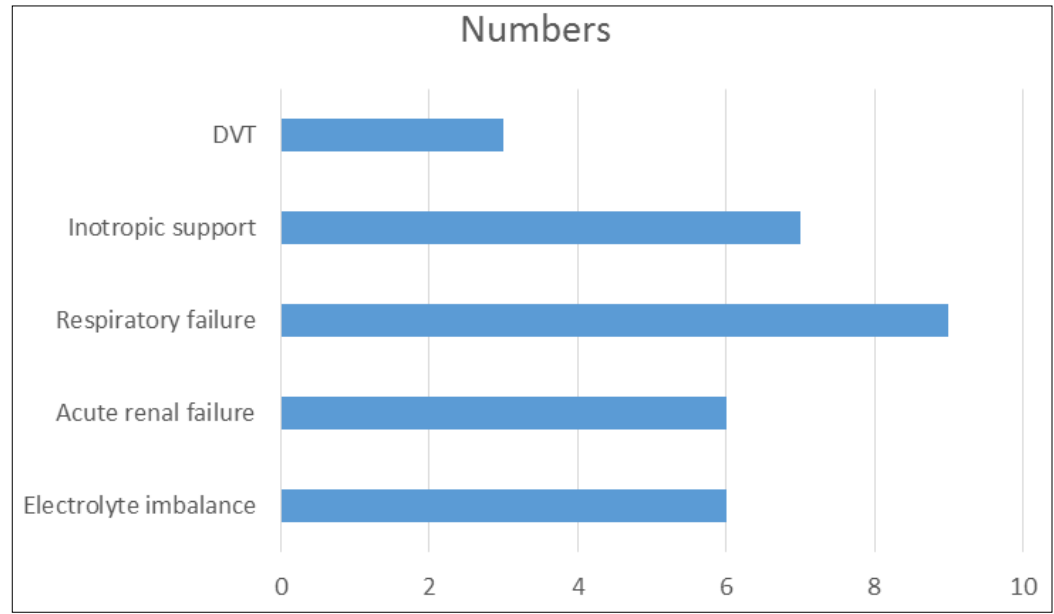

Fig 6: Reason for ICU admissions in the elective group

\section{Discussion}

The study was done to analyze the cause for intensive care in orthopaedic patients. The study showed that trauma and polytrauma patients were more likely to get complications leading to ICU care ${ }^{[3]}$. This is due to the acute disturbance in the metabolic, respiratory and cardiac and renal functions leading to rapid decompensation. The average age was 64 showing that geriatric population were more likely to develop life threatening complications due to associated comorbidities and decreased cardiac and respiratory reserve ${ }^{[5,9]}$.

Hip fractures especially in the geriatric group were more prone to develop complications leading to ICU management. These patients should be monitored closely and proactive measures should be taken to avoid deterioration of function [4].

Electrolyte disturbances were common in both trauma and elective group, especially in the elderly. Hyponatremia was a common cause, especially in the elderly and may due to the poor nutrition in general ${ }^{[11]}$.

Respiratory complications were also common. Baseline respiratory function should be done in all vulnerable patients to predict and proactively manage complications, if any in 
these patients ${ }^{[4,10]}$.

Inotropic support was needed in a significant proportion of polytrauma patients, especially due neurogenic shock.

\section{Conclusion}

With increasing trauma and polytrauma cases, coupled with increasing geriatric population, intensive care has becoming more and more common in orthopaedic patients. Elderly patients are more likely to develop complications due to associated comorbidities. Awareness of these possible complications and a proactive approach will help in decreasing the mortality and morbidity associated with it. A thorough understanding of comorbidities, possible intraoperative complications and early intervention is required. Such critical care may include-monitoring the patients closely, inotropic support, non invasive and invasive ventilation.

\section{References}

1. Suryagadham. Orthopaedic patients who require icu admissions. Journal of Trauma \& treatment 2013. Doi:10.4172/2167-1222.1000169

2. The Royal College of Surgeons of England and the British Orthopaedic Association. Better Care for the Severely Injured. London: RCSEND, Professional Standards and Regulation 2000.

3. Duane $\mathrm{T}$, et al. Are trauma patients better off in a traumaicu? Journal of emergencies, trauma and shock, 2008.

4. Taylor JM, et al. Critical care challenges in the orthopaedic patients, Critical Care Medicine: September 2006;34(9):S191-S199.

doi: 10.1097/01.CCM.0000231880.18476.D8

5. Romanmealmed. Perioperative medicine 5, Article 2016, 19.

6. Karaaslan, et al. Perioperativeanaethesia related complications-European journal of anaestheliology 2014;31:250.

7. Joy Steadman et al. Life threatening perioperative complications. Trauma surgery \& Acute care open 2017;2(1):e000113.

8. Perez Agaba et al. Comparison of postoperative complications after total hip arthroplasty among patients receiving aspirin, enoxaparin, warfarin, and factor $\mathrm{Xa}$ inhibitors-J Orthop. 2017;14(4):537-543. Published online 2017 Aug 14. doi: 10.1016/j.jor.2017.08.002

9. Jay R. Lieberman Vincent Cheng 1, Mark P Cote Pulmonary Embolism Rates Following Total Hip Arthroplasty With Prophylactic Anticoagulation: Some Pulmonary Emboli Cannot Be Avoided-J Arthroplasty 2017;32(3):980-986.doi: 10.1016/j.arth.2016.09.006.

10. Mohsin Khan 1, Craig Della Valle J, David Jacofsky J, Michael Meneghini R, Fares Haddad S. Early postoperative complications after total hip arthroplasty: current strategies for prevention and treatment-Instr Course Lectures 2015;64:337-46.

11. Antonelli Incalzi R1, Gemma A, Capparella O, Terranova L, Sanguinetti C, Carbonin PU. Post-operative electrolyte imbalance: its incidence and prognostic implications for elderly orthopaedic patients-Age Ageing 1993;22(5):325-31. doi: 10.1093/ageing/22.5.325. 